\title{
Validation of the Italian Version of the Test of Adherence to Inhalers
}

Baiardini $I^{1,2}$, Paoletti $\mathrm{G}^{1,2}$, Malipiero $\mathrm{G}^{1}$, Giua $\mathrm{C}^{3}$, Keber $\mathrm{E}^{3}$, Canonica $\mathrm{GW}^{1,2}$, Heffler $\mathrm{E}^{1,2}$

'Personalized Medicine, Asthma and Allergy, Humanitas Clinical and Research Center IRCCS, Rozzano, Italy

${ }^{2}$ Department of Biomedical Sciences, Humanitas University,

Pieve Emanuele, Italy

${ }^{3}$ Società Italiana Farmacia Clinica (SIFAC), Cagliari, Italy

J Investig Allergol Clin Immunol 2020; Vol. 30(6): 450-452

doi: 10.18176/jiaci.0536

Key words: Asthma. Adherence. Inhalers. Test of Adherence to Inhalers. Validation.

Palabras clave: Asma. Adherencia. Inhaladores. Test de adherencia a los inhaladores. Validación.

Effective management of asthma requires long-term adherence to inhaled drug therapies [1]. Regular use of maintenance treatment is associated with disease control, reduction in morbidity and costs, and, therefore, improvement in health-related quality of life [2]. Despite these benefits, nonadherence to asthma therapy is still frequent in clinical practice, ranging from $40 \%$ to $80 \%$ [3].

Although no gold standard exists, the various ways of assessing the level of adherence include laboratory values, pharmacy refill, electronic monitoring, and self-report questionnaires. These approaches are all characterized by strengths and limitations. In clinical practice, there is a need to assess adherence and improve the patient's engagement in shared decision-making and self-management. For a tool to be incorporated into the workflow of routine care, it must be valid, reliable, and inexpensive, as well as short and easy to complete, score, and interpret.

Most available self-reported measures of medication have been developed to assess patient's behavior independently of the drug or route of administration [4]. The Test of Adherence to Inhalers (TAI) [5] is the only tool for evaluation of adherence to inhalers that is tailored to patients with chronic obstructive pulmonary disease or asthma. It is composed of 12 items: the first 10 are completed by the patient and evaluate the level of adherence; in the remaining 2, health professionals are asked to detect 2 possible causes of unwitting nonadherent behavior (patient's knowledge of the prescribed regimen and inhaler technique). The questionnaire was originally developed in Spain [5] and is available in many languages. The original Spanish TAI proved to be a valid and reliable instrument [6], and its validity was recently confirmed in Farsi [7]. Here, we describe the psychometric properties of the Italian version of the TAI in terms of validity and reliability [8].

Adult asthmatic patients ( $\geq 18$ years) in treatment with inhaled therapy for at least 2 months and visiting community pharmacies that employed clinical pharmacy specialists were 
Table. Factors Identified Using Principal Components Analysis on a Full Data Set ${ }^{\mathrm{a}}$

\begin{tabular}{lcccc}
\hline & Factor 1 & Factor 2 & Factor 3 & Factor 4 \\
\hline Item 1 & 0.224 & $0.921^{\mathrm{a}}$ & 0.066 & 0.102 \\
Item 2 & 0.227 & $0.912^{\mathrm{a}}$ & 0.152 & 0.071 \\
Item 3 & $0.758^{\mathrm{a}}$ & 0.221 & 0.022 & 0.103 \\
Item 4 & $0.801^{\mathrm{a}}$ & 0.180 & 0.163 & 0.059 \\
Item 5 & $0.736^{\mathrm{a}}$ & 0.095 & 0.049 & -0.021 \\
Item 6 & $0.454^{\mathrm{a}}$ & 0.150 & 0.445 & 0.112 \\
Item 7 & 0.288 & 0.100 & $0.689^{\mathrm{a}}$ & 0.194 \\
Item 8 & $0.616^{\mathrm{a}}$ & 0.079 & 0.207 & 0.318 \\
Item 9 & 0.263 & 0.129 & 0.471 & $0.536^{\mathrm{a}}$ \\
Item 10 & -0.070 & 0.046 & $0.747^{\mathrm{a}}$ & -0.130 \\
Item 11 & 0.152 & 0.061 & -0.215 & $0.701^{\mathrm{a}}$ \\
Item 12 & -0.005 & 0.048 & 0.167 & $0.711^{\mathrm{a}}$ \\
\hline
\end{tabular}

${ }^{\mathrm{a}}$ Component for which each item scored highest.

asked to participate in a monitoring program. Patients were assessed at baseline and after 2 months. At the first visit, sociodemographic data, disease patterns, smoking habits, and current treatment were collected. At each visit, patients were administered the Italian version of the TAI and the Asthma Control Test (ACT) [9].

A factor analysis was also carried out to determine scale dimensionality. Convergent validity was assessed using the Spearman correlation coefficient to examine the relationships between TAI and ACT. The Cronbach $\alpha$ for the whole instrument was used to determine the internal consistency of the TAI. In order to ascertain the reliability of the TAI, we used the test-retest interclass coefficient (ICC) for each item and for the whole questionnaire.

The study population comprised 81 patients, 43 of whom were women (mean [SD] age, 53.6 [16.2] years; 22 smokers). Most patients had a university degree or higher diploma (54), and the remainder had completed secondary education (15) and primary school (12). The duration of asthma was $\leq 3$ months in 10 patients, from 3 to 36 months in 5 patients, and $>3$ years in 61 patients. A metered-dose inhaler was the principal device for 44 patients, and a dry powder inhaler was used by 37. The mean (SD) ACT score was 19.0 (4.5). Asthma was totally controlled in 7 patients, well controlled in 40 , and uncontrolled in 34.

Factor analysis revealed a 4-dimensional structure, which explained up to $70.08 \%$ of the total variance (Table). TAI scores showed a significant correlation with the ACT (Spearman correlation coefficient, 0.287; $P=.009$ ). The Cronbach $\alpha$ of 0.759 for the whole instrument exceeded the minimum internal consistency standard of $\geq 70$ recommended for group comparison. Moderate-good reproducibility was recorded for all items, with ICC values ranging from 0.17 to 0.85 . The reliability of the whole questionnaire was good, with an ICC of 7.6.
The results of our study confirm that the Italian version of the TAI meets the standards for good validity, internal consistency, and reliability. The factor analysis maintained the original distinction between items completed by the patient (items 1-10) and those completed by the health professionals (items 11 and 12). However, the first 10 items were grouped into 3 factors that refer to well-known subtypes of nonadherence. The first factor combined the items related to memory problems; the second factor grouped items with respect to the influence of subjective experiences; and the third factor encompassed the items that detected the presence of practical difficulties. Moreover, on the basis of the factor analysis results, only item 9 (assigned to the fourth factor) might have been allocated to another domain (factor 3). As expected for 2 instruments that provide a measure of different constructs, the correlation between TAI and ACT was low but significant, with the sign in the expected direction. The internal consistency of the TAI is good, with Cronbach $\alpha$ values that exceeded the recommended threshold [10]. While lower than the values seen for the original instrument and for the Farsi version, the ICC recorded indicates satisfactory reliability.

In conclusion, we confirmed that the Italian version of the TAI is a valid and reliable tool for identifying and monitoring nonadherence. Considering its psychometric characteristics, the questionnaire is suitable for use in both research and clinical practice in Italy. Future studies should aim to confirm our findings in larger cohorts. Moreover, it would be of considerable interest to explore the psychometric properties that have not yet been determined, namely, responsiveness and minimal detectable change in TAI scores, which may improve health outcomes.

\section{Funding}

The authors declare that no funding was received for the present study.

\section{Conflicts of Interest}

Dr Canonica reports grants and personal fees from Menarini, Alk Abelló, Anallergo, Boehringer Ingelheim, Chiesi, Circassia, Genentech, Guidotti Malesci, GSK, Hal Allergy, Meda, Merck, Merck Sharp \& Dome, Novartis, Recordati-InnuvaPharma, Roche, Sanofi, Stallergenes, UCB Pharma, Uriach Pharma, Teva, AstraZeneca, Thermo Fisher, Valeas, and Vibor Pharma outside the submitted work.

Dr Heffler reports personal fees from AstraZeneca, Sanofi, Novartis, Teva, GSK, Circassia, Boehringer Ingelheim, Valeas, and Nestlé Purina outside the submitted work.

The remaining authors declare that they have no conflicts of interest.

\section{References}

1. Global Initiative for Asthma (GINA), Global Strategy for Asthma Management and Prevention (Updated), www. ginasthma.org Accessed: April 2, 2020

2. Baiardini I, Novakova S, Mihaicuta S, Oguzulgen IK, Canonica GW. Adherence to treatment in allergic respiratory diseases. Expert Rev Respir Med. 2019;13(1):53-62. 
3. Tangirala NC, O'Conor R, Wolf MS, Wisnivesky JP, Federman AD. Validity of the Medication Adherence Rating Scale for Adherence to Inhaled Corticosteroids among Older Adults with Asthma or Chronic Obstructive Pulmonary Disease. COPD. 2020;17(1):74-80.

4. Plaza V. Update on questionnaires for assessing adherence to inhaler devices in respiratory patients. Curr Opin Allergy Clin Immunol. 2018;18(1):44-50.

5. Plaza V, Fernández-Rodríguez C, Melero C, Cosío BG, Entrenas $L M$, de Llano LP, et al. Validation of the 'test of the adherence to inhalers' (TAI) for asthma and COPD patients. J Aerosol Med Pulm Drug Deliv. 2016;29:142-52.

6. Urrutia I, Delgado J, Domínguez-Ortega J, Mascarós E, Pérez M, Resler G, et al; MISTRAL Investigators Group. Clinical Factors Associated With Overuse of Asthma Reliever Medication. J Investig Allergol Clin Immunol. 2020;30(1):42-8.

7. Khosravi S, Rafiei F, Norozy M, Khanmohamadi Hezave A, Ebrahimabadi M. Cross-Cultural Adaptation Of The Persian Version Of Test Of The Adherence To Inhalers (TAI). Patient Prefer Adherence. 2019;13:1693-9.

8. Sousa VD, Rojjanasrirat W. Translation, adaptation and validation of instruments or scales for use in cross-cultural health care research: a clear and user-friendly guideline. J Eval Clin Pract. 2011;17(2):268-74.

9. Nathan RA, Sorkness CA, Kosinski M, Schatz M, Li JT, Marcus $\mathrm{P}$, et al. Development of the asthma control test: a survey for assessing asthma control. J Allergy Clin Immunol. 2004;113:59-65.

10. Cronbach LJ. Coefficient alpha and the internal structure of tests. Psychometrika. 1951;16(3):297-334.

Manuscript received April 9, 2020; accepted for publication April 24, 2020.

Enrico Heffler

Personalized Medicine, Asthma and Allergy Istituto Clinico Humanitas IRCCS

Via Alessandro Manzoni 56

20089 - Rozzano (MI) - Italy

E-mail: enrico.heffler@hunimed.eu 